QUARTERLY OF APPLIED MATHEMATICS

VOLUME LXIX, NUMBER 1

MARCH 2011, PAGES 1-13

S 0033-569X(2010)01187-6

Article electronically published on December 9, 2010

\title{
ANALYTICITY OF TRANSMISSION PROBLEM TO THERMOELASTIC PLATES
}

\author{
BY \\ HUGO D. FERNÁNDEZ SARE (Department of Research and Development, National Laboratory for \\ Scientific Computation, Rua Getulio Vargas 333, Quitandinha CEP 25651-070, Petrópolis, Rio de \\ Janeiro-Brazil) \\ AND \\ JAIME E. MUÑOZ RIVERA (Department of Research and Development, National Laboratory for \\ Scientific Computation, Rua Getulio Vargas 333, Quitandinha CEP 25651-070, Petrópolis, Rio de \\ Janeiro-Brazil)
}

Abstract. In this paper we consider an oscillation model to a plate comprised of two different thermoelastic materials; that is, we study a transmission problem to thermoelastic plates. Our main result is to prove that the corresponding semigroup associated to this problem is of analytic type.

1. Introduction. In thermoelasticity, the classical linear model to thermoelastic plates is given by the following system:

$$
\begin{aligned}
\rho u_{t t}-\mu \Delta u_{t t}+\gamma \Delta^{2} u+\alpha \Delta \theta & =0 \quad \text { in } \quad \Omega \subset \mathbb{R}^{2}, \\
c \theta_{t}-\kappa \Delta \theta-\alpha \Delta u_{t}=0 & \text { in } \quad \Omega \subset \mathbb{R}^{2},
\end{aligned}
$$

where $\rho, \gamma, \alpha, c, \kappa$ and $\mu$ are positive constants. The physical model can be seen in 2 ] and the references therein. Several authors studied this system with different types of boundary conditions (Dirichlet, Neumann, clamped, etc.) and proved the exponential stability of the solutions; see for example [1, 7, 8, 11] and references therein. In the particular case $\mu=0$, it was proved in [3, 4, 5, that the semigroup associated to the above system is analytic. If $\mu>0$, the system has a hyperbolic character and hence the corresponding semigroup is not analytic, but the exponential stability of solutions is kept; see for example [7].

Received March 4, 2009.

2000 Mathematics Subject Classification. Primary 35B40, 74H40.

Key words and phrases. Thermoelastic plates, transmission problem, analytic semigroup.

H. D. Fernández Sare is supported by the CNPq grant 152398/2007-4. J. E. Muñoz Rivera is supported by the CNPq grant 309166/2007-1.

E-mail address: hugosare@lncc.br, hugo@im.ufrj.br

E-mail address: rivera@lncc.br

(C)2010 Brown University Reverts to public domain 28 years from publication 
On the other hand, in the case of transmission problems in thermoelasticity, the situation concerning exponential stability is more delicate and, to our best knowledge, there exist only a few results. For example, in [9], the authors proved that a partial thermoelastic dissipation is enough to stabilize the system exponentially. That is, they considered the model

$$
\begin{aligned}
\rho_{1} u_{t t}-\mu_{1} \Delta u_{t t}+\gamma_{1} \Delta^{2} u+\alpha_{1} \Delta \theta=0 & \text { in } \quad \Omega_{1}, \\
c \theta_{t}-\kappa \Delta \theta-\alpha \Delta u_{t}=0 & \text { in } \quad \Omega_{1}, \\
\rho_{2} v_{t t}-\mu_{2} \Delta v_{t t}+\gamma_{2} \Delta^{2} v=0 & \text { in } \quad \Omega_{2},
\end{aligned}
$$

where $\Omega=\Omega_{1} \cup \Omega_{2} \subset \mathbb{R}^{n}, \Omega_{1} \cap \bar{\Omega}_{2}=\emptyset$, with $\rho_{i}, \gamma_{i}, \alpha, c, \kappa>0, \mu \geq 0,(i=1,2)$ together with Dirichlet boundary conditions, suitable transmission conditions and of course initial conditions. Using multiplier techniques they proved the exponential stability of the solutions $u$ and $v$.

Returning to analytical results, as it is known to linear systems, analyticity is synonymous with asymptotic stability and smoothing effect property, which means that no matter how irregular the initial data is, the corresponding solution is of $C^{\infty}$-class, for any $t>0$. Then the question that arises is the following: Is it possible to obtain analytic semigroups associated to transmission problems in thermoelasticity? To our best knowledge, there are no results about this matter in the literature and to fill this gap we study this point here.

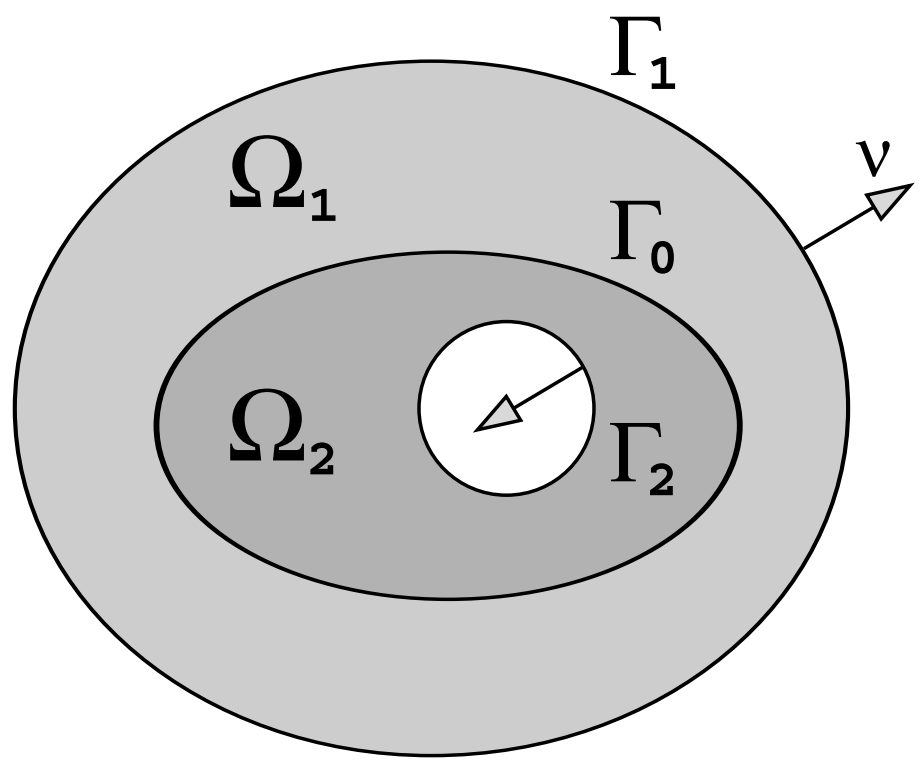

FIG. 1

In other words, we consider a transmission problem associated to thermoelastic plates, which can be seen as an evolution system with discontinuous coefficients. With this in mind, we cannot expect $C^{\infty}$ solutions on the whole domain $\Omega$, for $t>0$. More 
precisely, the transmission problem to thermoelastic plates that we consider here, over each component of $\Omega=\Omega_{1} \cup \Omega_{2}$, can be written as

$$
\begin{array}{rll}
\rho_{1} u_{t t}+\gamma_{1} \Delta^{2} u+\alpha_{1} \Delta \theta=0 & \text { in } \quad \Omega_{1} \\
c_{1} \theta_{t}-\kappa_{1} \Delta \theta-\alpha_{1} \Delta u_{t}=0 & \text { in } \quad \Omega_{1} \\
\rho_{2} v_{t t}+\gamma_{2} \Delta^{2} v+\alpha_{2} \Delta \omega=0 & \text { in } \quad \Omega_{2} \\
c_{2} \omega_{t}-\kappa_{2} \Delta \omega-\alpha_{2} \Delta v_{t}=0 & \text { in } \quad \Omega_{2}
\end{array}
$$

with boundary conditions

$$
u=\frac{\partial u}{\partial \nu}=0 \quad \text { in } \quad \Gamma_{1} \quad v=\frac{\partial v}{\partial \nu}=0 \quad \text { in } \quad \Gamma_{2}
$$

and transmission conditions

$$
\begin{gathered}
u=v, \quad \frac{\partial u}{\partial \nu}=\frac{\partial v}{\partial \nu} \quad \text { in } \quad \Gamma_{0} \\
\theta=\omega, \quad \kappa_{1} \frac{\partial \theta}{\partial \nu}+\alpha_{1} \frac{\partial u_{t}}{\partial \nu}=\kappa_{2} \frac{\partial \omega}{\partial \nu}+\alpha_{2} \frac{\partial v_{t}}{\partial \nu} \quad \text { in } \quad \Gamma_{0} \\
\gamma_{1} \Delta u=\gamma_{2} \Delta v, \quad \gamma_{1} \frac{\partial \Delta u}{\partial \nu}+\alpha_{1} \frac{\partial \theta}{\partial \nu}=\gamma_{2} \frac{\partial \Delta v}{\partial \nu}+\alpha_{2} \frac{\partial \omega}{\partial \nu} \quad \text { in } \quad \Gamma_{0} .
\end{gathered}
$$

The initial conditions are given by

$$
\begin{aligned}
& u(\cdot, 0)=u_{0}, \quad u_{t}(\cdot, 0)=u_{1}, \quad \theta(\cdot, 0)=\theta_{0}, \quad \text { in } \quad \Omega_{1}, \\
& v(\cdot, 0)=v_{0}, \quad v_{t}(\cdot, 0)=v_{1}, \quad \omega(\cdot, 0)=\omega_{0}, \quad \text { in } \quad \Omega_{2},
\end{aligned}
$$

where $\rho_{i}, \gamma_{i}, \alpha_{i}, c_{i}, \kappa_{i}$ stand as positive constants $(i=1,2)$. The domain $\Omega=\Omega_{1} \cup \Omega_{2}$ is a bounded open set in $\mathbb{R}^{2}$ with boundary $\partial \Omega=\Gamma_{1} \cup \Gamma_{2}$ where $\Gamma_{1}, \Gamma_{2}$ are two smooth curves such that $\bar{\Gamma}_{1} \cap \bar{\Gamma}_{2}=\emptyset$ and $\nu$ is the unit normal vector outside of $\Omega$. Let us denote by $\Gamma_{0}$ the common smooth curve between $\Omega_{1}$ and $\Omega_{2}$.

The main result of this paper is to prove that the semigroup associated to system (1.6)-(1.15) is analytic and, by direct consequence of this result, the solutions $u$ and $v$ of this system are exponentially stable when the time goes to infinity; they also have the smoothing effect property over $\Omega_{1}$ and $\Omega_{2}$ respectively, but not over the whole domain $\Omega$.

It is important to emphasize here that the main contribution of this paper is to connect solutions of a thermoelastic transmission problem (discontinuous in principle because we have two different materials to $\Omega=\Omega_{1} \cup \Omega_{2}$ ), with analytic semigroups which gives us the regularity of the solutions $u$ and $v$ in each sub-domain $\Omega_{1}$ and $\Omega_{2}$ respectively, where the solutions are defined. Of course, from the theory of analytic semigroups, we deduce directly the exponential stability of the solutions of our system, but the case where only one region includes the thermoelastic coupling (for example, only in $\Omega_{1}$ ) is the most interesting to be considered and was solved satisfactorily by the authors Muñoz Rivera, J. E. and Portillo Oquendo, H. in 9 .

This paper is organized as follows. In section 2 we establish the semigroup formulation associated to system (1.6)-(1.15) as well as the main tools used in the next sections. In 
section 3 we establish and prove the main result of this paper; that is, we prove that the semigroup associated to system (1.6)-(1.15) is analytic.

2. Notations and semigroup formulation. Here we establish the notations and main tools that will be used in the next sections. First, we define the Hilbert spaces

$$
\begin{aligned}
\mathbb{H}^{m}(\Omega) & :=H^{m}\left(\Omega_{1}\right) \times H^{m}\left(\Omega_{2}\right) \quad(m \geq 1), \\
\mathbb{H}^{0}(\Omega) & :=L^{2}\left(\Omega_{1}\right) \times L^{2}\left(\Omega_{2}\right)
\end{aligned}
$$

and

$$
\begin{aligned}
& \mathbb{H}_{\Gamma}^{m}(\Omega):=\left\{\left(\phi_{1}, \phi_{2}\right) \in \mathbb{H}^{m}(\Omega) \quad: \quad \phi_{i}=0 \quad \text { in } \quad \Gamma_{i}(i=1,2) \quad\right. \text { and } \\
&\left.\frac{\partial^{k} \phi_{1}}{\partial \nu}=\frac{\partial^{k} \phi_{2}}{\partial \nu} \quad \text { in } \quad \Gamma_{0}, k=0,1, \ldots,(m-1)\right\} \quad(m \geq 1) .
\end{aligned}
$$

Using these notations, let us define an appropriate Hilbert space where the semigroup will be defined, which is

$$
\mathcal{H}=\mathbb{H}_{\Gamma}^{2}(\Omega) \times \mathbb{H}^{0}(\Omega) \times \mathbb{H}^{0}(\Omega),
$$

with associated norm to $U=(u, v, z, y, \theta, \omega)^{\prime} \in \mathcal{H}$ defined by

$$
\begin{aligned}
\|U\|_{\mathcal{H}}^{2}= & \gamma_{1}\|\Delta u\|_{L^{2}\left(\Omega_{1}\right)}^{2}+\gamma_{2}\|\Delta v\|_{L^{2}\left(\Omega_{2}\right)}^{2}+\rho_{1}\|z\|_{L^{2}\left(\Omega_{1}\right)}^{2}+\rho_{2}\|y\|_{L^{2}\left(\Omega_{2}\right)}^{2} \\
& +c_{1}\|\theta\|_{L^{2}\left(\Omega_{1}\right)}^{2}+c_{2}\|\omega\|_{L^{2}\left(\Omega_{2}\right)}^{2} .
\end{aligned}
$$

Also, we define the linear operator

$$
\mathcal{A}\left(\begin{array}{l}
u \\
v \\
z \\
y \\
\theta \\
\omega
\end{array}\right):=\left(\begin{array}{c}
z \\
y \\
-\gamma_{1} \Delta^{2} u-\alpha_{1} \Delta \theta \\
-\gamma_{2} \Delta^{2} v-\alpha_{2} \Delta \omega \\
\kappa_{1} \Delta \theta+\alpha_{1} \Delta z \\
\kappa_{2} \Delta \omega+\alpha_{2} \Delta y
\end{array}\right)
$$

over the domain $D(\mathcal{A}) \subset \mathcal{H}$ defined by

$$
\begin{aligned}
& D(\mathcal{A}):=\left\{U=(u, v, z, y, \theta, \omega)^{\prime} \in \mathcal{H}:(u, v) \in \mathbb{H}^{4}(\Omega) \cap \mathbb{H}_{\Gamma}^{2}(\Omega), \quad(z, y) \in \mathbb{H}_{\Gamma}^{2}(\Omega)\right. \\
& (\theta, \omega) \in \mathbb{H}^{2}(\Omega) \cap \mathbb{H}_{\Gamma}^{1}(\Omega), \quad\left(\gamma_{1} \Delta u, \gamma_{2} \Delta v\right) \in \mathbb{H}_{\Gamma}^{1}(\Omega) \\
& \kappa_{1} \frac{\partial \theta}{\partial \nu}+\alpha_{1} \frac{\partial z}{\partial \nu}=\kappa_{2} \frac{\partial \omega}{\partial \nu}+\alpha_{2} \frac{\partial y}{\partial \nu} \quad \text { in } \quad \Gamma_{0} \quad \text { and } \\
& \left.\gamma_{1} \frac{\partial \Delta u}{\partial \nu}+\alpha_{1} \frac{\partial \theta}{\partial \nu}=\gamma_{2} \frac{\partial \Delta v}{\partial \nu}+\alpha_{2} \frac{\partial \omega}{\partial \nu} \quad \text { in } \quad \Gamma_{0}\right\},
\end{aligned}
$$


which is associated to system (1.6)-(1.10) with the classical notations $z:=u_{t}$ and $y:=v_{t}$. It is not difficult to prove that the operator $\mathcal{A}$ is dissipative and

$$
0 \in \rho(\mathcal{A}) .
$$

Therefore, using semigroups theory (see [6, 10]), we have the following theorem.

Theorem 2.1. The linear operator $\mathcal{A}$ given by (2.2) and associated to system (1.6)-(1.10) is the infinitesimal generator of a $C_{0}$-semigroup of contractions on the Hilbert space $\mathcal{H}$ defined by (2.1).

On the other hand, the main tool to prove the analyticity of the semigroup generated by $\mathcal{A}$ is the following well-known theorem

Theorem 2.2. A semigroup of contractions $\left\{\mathrm{e}^{t \mathcal{A}}\right\}_{t \geq 0}$ in a Hilbert space $\mathcal{H}$ with norm $\|\cdot\|_{\mathcal{H}}$ is of analytic type if and only if

$$
i \mathbb{R} \subset \varrho(\mathcal{A})
$$

and

$$
\lim _{|\lambda| \rightarrow \infty}\left\|\lambda(i \lambda I-\mathcal{A})^{-1}\right\|_{\mathcal{L}(\mathcal{H})}<\infty .
$$

Proof. See, e.g., [6, Theorem 1.3.2].

In order to apply Theorem 2.2 we will analyze the solution of the resolvent equation

$$
(i \lambda I-\mathcal{A}) U=F \quad \text { in } \quad \mathcal{H}
$$

which, in terms of its components, can be written as

$$
\begin{array}{rlrl}
i \lambda u-z & =f^{1} & \text { in } H^{2}\left(\Omega_{1}\right) \\
i \lambda v-y & =f^{2} & \text { in } H^{2}\left(\Omega_{2}\right) \\
i \lambda \rho_{1} z+\gamma_{1} \Delta^{2} u+\alpha_{1} \Delta \theta & =\rho_{1} f^{3} & \text { in } & L_{2}\left(\Omega_{1}\right) \\
i \lambda \rho_{2} y+\gamma_{2} \Delta^{2} v+\alpha_{2} \Delta \omega & =\rho_{2} f^{4} & \text { in } & L_{2}\left(\Omega_{2}\right) \\
i \lambda c_{1} \theta-\kappa_{1} \Delta \theta-\alpha_{1} \Delta z & =c_{1} f^{5} & \text { in } & L_{2}\left(\Omega_{1}\right) \\
i \lambda c_{2} \omega-\kappa_{2} \Delta \omega-\alpha_{2} \Delta y & =c_{2} f^{6} & \text { in } & L_{2}\left(\Omega_{2}\right) .
\end{array}
$$

Our starting point to arrive at the hypotheses of Theorem 2.2 is to use the estimate given by the thermal dissipation, which is given by the following Lemma.

Lemma 2.3. Let $U=(u, v, z, y, \theta, \omega)^{\prime} \in D(\mathcal{A})$ be a solution of the system (2.6)-(2.11) with $F=\left(f^{1}, f^{2}, f^{3}, f^{4}, f^{5}, f^{6}\right)^{\prime} \in \mathcal{H}$. Then

$$
\kappa_{1}\|\nabla \theta\|_{L^{2}\left(\Omega_{1}\right)}^{2}+\kappa_{2}\|\nabla \omega\|_{L^{2}\left(\Omega_{2}\right)}^{2} \leq C\|U\|_{\mathcal{H}}\|F\|_{\mathcal{H}},
$$

where $C$ is a positive constant independent of $\lambda$ and $U \in D(\mathcal{A})$.

Proof. By multiplying (2.5) by $U \in \mathcal{H}$ and taking the real part, our conclusion follows. 
3. Analyticity. In order to prove our main result, note that to show the condition (2.4) from Theorem 2.2 is equivalent to stating that there exists a constant $C>0$ such that

$$
\|U\|_{\mathcal{H}}=\left\|(i \lambda I-\mathcal{A})^{-1} F\right\|_{\mathcal{H}} \leq \frac{C}{|\lambda|}\|F\|_{\mathcal{H}}, \quad \forall|\lambda| \geq \lambda_{0}>0,
$$

or state that for any $\varepsilon>0$, there exists $C_{\varepsilon}>0$ such that

$$
|\lambda|^{2}\|U\|_{\mathcal{H}}^{2} \leq \varepsilon|\lambda|^{2}\|U\|_{\mathcal{H}}^{2}+C_{\varepsilon}\|F\|_{\mathcal{H}}^{2}, \quad \forall|\lambda| \geq \lambda_{0}>0
$$

This late relation will be established as a consequence of the following Propositions 3.13 .3 and 3.4

Proposition 3.1. Let $U=(u, v, z, y, \theta, \omega)^{\prime} \in D(\mathcal{A})$ be a solution of the system (2.6)(2.11) with $F=\left(f^{1}, f^{2}, f^{3}, f^{4}, f^{5}, f^{6}\right)^{\prime} \in \mathcal{H}$. Then, for any $\varepsilon>0$, there exists $C_{\varepsilon}>0$ such that

$$
|\lambda|^{2}\|\theta\|_{L^{2}\left(\Omega_{1}\right)}^{2}+|\lambda|^{2}\|\omega\|_{L^{2}\left(\Omega_{2}\right)}^{2} \leq \varepsilon|\lambda|^{2}\|U\|_{\mathcal{H}}^{2}+C_{\varepsilon}\|F\|_{\mathcal{H}}^{2} .
$$

Proof. Multiplying equation (2.10) by $i \lambda \theta$ in $L^{2}\left(\Omega_{1}\right)$ and (2.11) by $i \lambda \omega$ in $L^{2}\left(\Omega_{2}\right)$, we obtain

$$
\begin{array}{r}
|\lambda|^{2} c_{1}\|\theta\|_{L^{2}\left(\Omega_{1}\right)}^{2}-i \kappa_{1} \lambda \int_{\Gamma_{0}} \frac{\partial \theta}{\partial \nu} \bar{\theta} d \Gamma-i \kappa_{1} \lambda\|\nabla \theta\|_{L^{2}\left(\Omega_{1}\right)}^{2}-i \alpha_{1} \lambda \int_{\Gamma_{0}} \frac{\partial z}{\partial \nu} \bar{\theta} d \Gamma \\
-i \alpha_{1} \lambda \int_{\Omega_{1}} \nabla z \nabla \bar{\theta} d x d y=-i \lambda c_{1} \int_{\Omega_{1}} f^{5} \bar{\theta} d x d y
\end{array}
$$

and

$$
\begin{array}{r}
|\lambda|^{2} c_{2}|| \omega\left\|_{L^{2}\left(\Omega_{2}\right)}^{2}-i \kappa_{2} \lambda \int_{\Gamma_{0}} \frac{\partial \omega}{\partial \nu} \bar{\omega} d \Gamma-i \kappa_{2} \lambda|| \nabla \omega\right\|_{L^{2}\left(\Omega_{2}\right)}^{2}-i \alpha_{2} \lambda \int_{\Gamma_{0}} \frac{\partial y}{\partial \nu} \bar{\omega} d \Gamma \\
-i \alpha_{1} \lambda \int_{\Omega_{2}} \nabla y \nabla \bar{\omega} d x d y=-i \lambda c_{1} \int_{\Omega_{2}} f^{6} \bar{\omega} d x d y
\end{array}
$$

respectively. Therefore, adding the last two equalities and using the transmission conditions (1.11)-(1.13), we have

$$
\begin{aligned}
|\lambda|^{2}\left[c_{1}\|\theta\|_{L^{2}\left(\Omega_{1}\right)}^{2}+c_{2}\|\omega\|_{L^{2}\left(\Omega_{2}\right)}^{2}\right] \leq & C|\lambda|\|U\|_{\mathcal{H}}\|F\|_{\mathcal{H}}+\alpha_{1}|\lambda|\|\nabla z\|_{L^{2}\left(\Omega_{1}\right)}\|\nabla \theta\|_{L^{2}\left(\Omega_{1}\right)} \\
& +\alpha_{2} \mid \lambda\|\| \nabla y\left\|_{L^{2}\left(\Omega_{2}\right)}\right\| \nabla \omega \|_{L^{2}\left(\Omega_{2}\right)} .
\end{aligned}
$$

To complete the proof we will now estimate $\|\nabla z\|_{L^{2}\left(\Omega_{1}\right)}$ and $\|\nabla y\|_{L^{2}\left(\Omega_{2}\right)}$ in (3.2). In fact, from (2.6) and (2.7) we get

$$
\begin{aligned}
\|z\|_{H^{2}\left(\Omega_{1}\right)} & \leq|\lambda|\|u\|_{H^{2}\left(\Omega_{1}\right)}+\left\|f^{1}\right\|_{H^{2}\left(\Omega_{1}\right)} \\
\|y\|_{H^{2}\left(\Omega_{2}\right)} & \leq|\lambda|\|v\|_{H^{2}\left(\Omega_{2}\right)}+\left\|f^{2}\right\|_{H^{2}\left(\Omega_{2}\right)} .
\end{aligned}
$$

On the other hand, using Gagliardo-Nirenberg inequalities we have

$$
\begin{aligned}
\|z\|_{H^{1}\left(\Omega_{1}\right)} & \leq C\|z\|_{H^{2}\left(\Omega_{1}\right)}^{1 / 2}\|z\|_{L^{2}\left(\Omega_{1}\right)}^{1 / 2} \\
\|y\|_{H^{1}\left(\Omega_{2}\right)} & \leq C\|y\|_{H^{2}\left(\Omega_{2}\right)}^{1 / 2}\|y\|_{L^{2}\left(\Omega_{2}\right)}^{1 / 2} .
\end{aligned}
$$


Then, applying (3.3) into (3.5) and (3.4) into (3.6) respectively, we deduce the following estimates:

$$
\begin{aligned}
\|z\|_{H^{1}\left(\Omega_{1}\right)} & \leq C|\lambda|^{1 / 2}\|U\|_{\mathcal{H}}+C\|U\|_{\mathcal{H}}^{1 / 2}\|F\|_{\mathcal{H}}^{1 / 2} \\
\|y\|_{H^{1}\left(\Omega_{1}\right)} & \leq C|\lambda|^{1 / 2}\|U\|_{\mathcal{H}}+C\|U\|_{\mathcal{H}}^{1 / 2}\|F\|_{\mathcal{H}}^{1 / 2} .
\end{aligned}
$$

Finally, using (3.7) and Lemma 2.3, for each $\varepsilon>0$ we have

$$
\begin{aligned}
\alpha_{1}|\lambda|\|\nabla z\|_{L^{2}\left(\Omega_{1}\right)}|| \nabla \theta \|_{L^{2}\left(\Omega_{1}\right)} & \leq \frac{\varepsilon}{16 C^{2}}|\lambda|\|z\|_{H^{1}\left(\Omega_{1}\right)}^{2}+C_{\varepsilon} \mid \lambda\|\| \nabla \theta \|_{H^{1}\left(\Omega_{1}\right)}^{2} \\
& \leq \frac{\varepsilon}{8}|\lambda|^{2}\|U\|_{\mathcal{H}}^{2}+C_{\varepsilon}|\lambda|\|U\|_{\mathcal{H}}\|F\|_{\mathcal{H}} \\
& \leq \frac{\varepsilon}{4}|\lambda|^{2}\|U\|_{\mathcal{H}}^{2}+C_{\varepsilon}\|F\|_{\mathcal{H}}^{2} ;
\end{aligned}
$$

this is

$$
\alpha_{1}|\lambda|\|\nabla z\|_{L^{2}\left(\Omega_{1}\right)}|| \nabla \theta\left\|_{L^{2}\left(\Omega_{1}\right)} \leq \frac{\varepsilon}{4}|\lambda|^{2}\right\| U\left\|_{\mathcal{H}}^{2}+C_{\varepsilon}\right\| F \|_{\mathcal{H}}^{2} .
$$

Similarly, using (3.8) and Lemma 2.3 we have

$$
\alpha_{2}|\lambda||| \nabla y\left\|_{L^{2}\left(\Omega_{2}\right)}|| \nabla \omega\right\|_{L^{2}\left(\Omega_{2}\right)} \leq \frac{\varepsilon}{4}|\lambda|^{2}|| U\left\|_{\mathcal{H}}^{2}+C_{\varepsilon}\right\| F \|_{\mathcal{H}}^{2} .
$$

Therefore, applying (3.9)-(3.10) into (3.2) our result follows.

As a consequence of Proposition 3.1 we now formulate and prove the following lemma, which will play an important role in the proof of Proposition 3.3 .

Lemma 3.2. With the same hypotheses as in Proposition 3.1, for any $\varepsilon>0$ and for any $|\lambda| \geq 1$, the following inequality

$$
\|\nabla z\|_{L^{2}\left(\Omega_{1}\right)}^{2}+\|\nabla y\|_{L^{2}\left(\Omega_{2}\right)}^{2} \leq \varepsilon C \mid \lambda\|\| U\left\|_{\mathcal{H}}^{2}+C_{\varepsilon} \frac{1}{|\lambda|}\right\| F \|_{\mathcal{H}}^{2}
$$

holds.

Proof. First, replacing $\varepsilon>0$ by $\varepsilon^{2}>0$ in Proposition 3.1, we have

$$
|\lambda|\|\theta\|_{L^{2}\left(\Omega_{1}\right)}^{2}+|\lambda|\|\omega\|_{L^{2}\left(\Omega_{2}\right)}^{2} \leq \varepsilon^{2}|\lambda|\|U\|_{\mathcal{H}}^{2}+C_{\varepsilon} \frac{1}{|\lambda|}\|F\|_{\mathcal{H}}^{2}
$$

for any $|\lambda| \geq 1$. On the other hand, multiplying (2.10) by $z$ in $L^{2}\left(\Omega_{1}\right)$ and (2.11) by $y$ in $L^{2}\left(\Omega_{2}\right)$ respectively, we obtain

$$
\begin{array}{r}
i \lambda c_{1} \int_{\Omega_{1}} \theta \bar{z} d x d y+\int_{\Gamma_{0}}\left[\kappa_{1} \frac{\partial \theta}{\partial \nu}+\alpha_{1} \frac{\partial z}{\partial \nu}\right] \bar{z} d \Gamma+\kappa_{1} \int_{\Omega_{1}} \nabla \theta \nabla \bar{z} d x d y \\
+\alpha_{1}\|\nabla z\|_{L^{2}\left(\Omega_{1}\right)}^{2}=c_{1} \int_{\Omega_{1}} f^{5} \bar{z} d x d y
\end{array}
$$


and

$$
\begin{array}{r}
i \lambda c_{2} \int_{\Omega_{2}} \omega \bar{y} d x d y-\int_{\Gamma_{0}}\left[\kappa_{2} \frac{\partial \omega}{\partial \nu}+\alpha_{2} \frac{\partial y}{\partial \nu}\right] \bar{y} d \Gamma+\kappa_{2} \int_{\Omega_{2}} \nabla \omega \nabla \bar{y} d x d y \\
+\alpha_{2}\|\nabla y\|_{L^{2}\left(\Omega_{2}\right)}^{2}=c_{2} \int_{\Omega_{2}} f^{6} \bar{y} d x d y
\end{array}
$$

respectively. Now we will estimate some terms of (3.12) and (3.13). In fact, by Lemma 2.3. we have the following inequalities:

$$
\begin{aligned}
\left|\kappa_{1} \int_{\Omega_{1}} \nabla \theta \nabla \bar{z} d x d y\right| & \leq C|| U\left\|_{\mathcal{H}}\right\| F\left\|_{\mathcal{H}}+\frac{\alpha_{1}}{3}\right\| \nabla z \|_{L^{2}\left(\Omega_{1}\right)}^{2} \\
& \leq \varepsilon|\lambda|\|U\|_{\mathcal{H}}^{2}+C_{\varepsilon} \frac{1}{|\lambda|}\|F\|_{\mathcal{H}}^{2}+\frac{\alpha_{1}}{3}\|\nabla z\|_{L^{2}\left(\Omega_{1}\right)}^{2}
\end{aligned}
$$

for any $|\lambda| \geq 1$. This is

$$
\left|\kappa_{1} \int_{\Omega_{1}} \nabla \theta \nabla \bar{z} d x d y\right| \leq \varepsilon|\lambda|\|U\|_{\mathcal{H}}^{2}+C_{\varepsilon} \frac{1}{|\lambda|}\|F\|_{\mathcal{H}}^{2}+\frac{\alpha_{1}}{3}\|\nabla z\|_{L^{2}\left(\Omega_{1}\right)}^{2} .
$$

Analogously we have

$$
\left|\kappa_{2} \int_{\Omega_{2}} \nabla \omega \nabla \bar{y} d x d y\right| \leq \varepsilon|\lambda|\|U\|_{\mathcal{H}}^{2}+C_{\varepsilon} \frac{1}{|\lambda|}\|F\|_{\mathcal{H}}^{2}+\frac{\alpha_{2}}{3}\|\nabla y\|_{L^{2}\left(\Omega_{1}\right)}^{2}
$$

for any $|\lambda| \geq 1$. Also, applying (3.11) we deduce that

$$
\begin{aligned}
\left|i \lambda c_{1} \int_{\Omega_{1}} \theta \bar{z} d x d y\right| & \leq \frac{1}{\varepsilon}|\lambda|\|\theta\|_{L^{2}\left(\Omega_{1}\right)}^{2}+\varepsilon|\lambda|\|z\|_{L^{2}\left(\Omega_{1}\right)}^{2} \\
& \leq \varepsilon|\lambda|\|U\|_{\mathcal{H}}^{2}+C_{\varepsilon} \frac{1}{|\lambda|}\|F\|_{\mathcal{H}}^{2}+\varepsilon|\lambda|\|U\|_{\mathcal{H}}^{2} ;
\end{aligned}
$$

this is

$$
\left|i \lambda c_{1} \int_{\Omega_{1}} \theta \bar{z} d x d y\right| \leq 2 \varepsilon|\lambda|\|U\|_{\mathcal{H}}^{2}+C_{\varepsilon} \frac{1}{|\lambda|}\|F\|_{\mathcal{H}}^{2}
$$

Similarly we get

$$
\left|i \lambda c_{2} \int_{\Omega_{2}} \omega \bar{y} d x d y\right| \leq 2 \varepsilon|\lambda|\|U\|_{\mathcal{H}}^{2}+C_{\varepsilon} \frac{1}{|\lambda|}\|F\|_{\mathcal{H}}^{2}
$$

Therefore, adding the equalities (3.12)-(3.13), using the transmission conditions (1.11)(1.13) and estimates (3.14)-(3.17), our conclusion follows immediately.

The aim of the next proposition is to obtain estimates to $|\lambda|\|z\|_{L^{2}\left(\Omega_{1}\right)}$ and $|\lambda|\|\omega\|_{L^{2}\left(\Omega_{2}\right)}$. As mentioned previously, the key tool is Lemma 3.2. more precisely, that Lemma will be used in the proof of inequality (3.27).

Proposition 3.3. Let $U=(u, v, z, y, \theta, \omega)^{\prime} \in D(\mathcal{A})$ be a solution of the system (2.6)(2.11) with $F=\left(f^{1}, f^{2}, f^{3}, f^{4}, f^{5}, f^{6}\right)^{\prime} \in \mathcal{H}$. Then, for any $\varepsilon>0$ there exists $C_{\varepsilon}>0$ such that

$$
|\lambda|^{2}\|z\|_{L^{2}\left(\Omega_{1}\right)}^{2}+|\lambda|^{2}\|y\|_{L^{2}\left(\Omega_{2}\right)}^{2} \leq \varepsilon C|\lambda|^{2}\|U\|_{\mathcal{H}}^{2}+C_{\varepsilon}\|F\|_{\mathcal{H}}^{2}
$$


Proof. First we set $z=z_{1}+z_{2}$ and $y=y_{1}+y_{2}$, where $z_{1}, y_{1}$ satisfy

$$
\begin{aligned}
& i \lambda \rho_{1} z_{1}-\Delta z_{1}=\rho_{1} f^{3} \text { in } \Omega_{1} \\
& i \lambda \rho_{2} y_{1}-\Delta y_{1}=\rho_{2} f^{4} \text { in } \Omega_{2}
\end{aligned}
$$

with boundary-transmission conditions

$$
\begin{gathered}
z_{1}=\frac{\partial z_{1}}{\partial \nu}=0 \quad \text { in } \quad \Gamma_{1}, \quad y_{1}=\frac{\partial y_{1}}{\partial \nu}=0 \quad \text { in } \quad \Gamma_{2}, \\
z_{1}=y_{1} \quad \text { in } \quad \Gamma_{0}, \quad \frac{\partial z_{1}}{\partial \nu}=\frac{\partial y_{1}}{\partial \nu} \quad \text { in } \quad \Gamma_{0},
\end{gathered}
$$

and hence $z_{2}, y_{2}$ satisfy

$$
\begin{aligned}
& i \lambda \rho_{1} z_{2}=-\gamma_{1} \Delta^{2} u-\Delta z_{1}-\alpha_{1} \Delta \theta \\
& i \lambda \rho_{2} y_{2}=-\gamma_{2} \Delta^{2} v-\Delta y_{1}-\alpha_{2} \Delta \omega .
\end{aligned}
$$

From $(\underline{3.19})-(\underline{3.20})$ we deduce that

$$
\begin{aligned}
|\lambda|\left\|z_{1}\right\|_{L^{2}\left(\Omega_{1}\right)}+\left\|z_{1}\right\|_{H^{2}\left(\Omega_{1}\right)} & \leq C\left\|f^{3}\right\|_{L^{2}\left(\Omega_{1}\right)} \\
|\lambda|\left\|y_{1}\right\|_{L^{2}\left(\Omega_{2}\right)}+\left\|y_{1}\right\|_{H^{2}\left(\Omega_{2}\right)} & \leq C\left\|f^{4}\right\|_{L^{2}\left(\Omega_{2}\right)},
\end{aligned}
$$

which implies that $z_{1}, y_{1}$ satisfy inequality (3.18). Therefore, to complete the proof, it is only necessary to verify that $z_{2}, y_{2}$ also satisfy inequality (3.18). In fact, from (3.21)-(3.22) we find

$$
\begin{aligned}
|\lambda|\left\|z_{2}\right\|_{H^{-2}\left(\Omega_{1}\right)} \leq C\left[\|\Delta u\|_{L^{2}\left(\Omega_{1}\right)}+\left\|z_{1}\right\|_{L^{2}\left(\Omega_{1}\right)}+\|\theta\|_{L^{2}\left(\Omega_{1}\right)}\right] \\
|\lambda|\left\|y_{2}\right\|_{H^{-2}\left(\Omega_{2}\right)} \leq C\left[\|\Delta v\|_{L^{2}\left(\Omega_{2}\right)}+\left\|y_{1}\right\|_{L^{2}\left(\Omega_{2}\right)}+\|\omega\|_{L^{2}\left(\Omega_{2}\right)}\right] .
\end{aligned}
$$

Then, using inequalities (3.23)-(3.24), we deduce that

$$
|\lambda|\left\|z_{2}\right\|_{H^{-2}\left(\Omega_{1}\right)}+|\lambda|\left\|y_{2}\right\|_{H^{-2}\left(\Omega_{2}\right)} \leq C|| U\left\|_{\mathcal{H}}+\frac{C}{|\lambda|}\right\| F \|_{\mathcal{H}}
$$

The next steps are directed to obtain estimates to $z_{1}, y_{1}$ in $L^{2}\left(\Omega_{1}\right), L^{2}\left(\Omega_{2}\right)$. To this aim we will establish estimates in the $H^{-1}$ and $H^{1}$ norms, and we will apply interpolation to obtain the desired $L^{2}$-estimates.

In fact, first note that by applying interpolation results, estimates (3.23)-(3.24) lead to

$$
\left\|z_{1}\right\|_{H^{1}\left(\Omega_{1}\right)}+\left\|y_{1}\right\|_{H^{1}\left(\Omega_{2}\right)} \leq C \frac{1}{|\lambda|^{1 / 2}}\|F\|_{\mathcal{H}}
$$


Then, applying interpolation again, from estimate (3.26) and Lemma 3.2 we deduce

$$
\begin{aligned}
\left\|z_{2}\right\|_{H^{-1}\left(\Omega_{1}\right)} & \leq C\left\|z_{2}\right\|_{H^{-2}\left(\Omega_{1}\right)}^{2 / 3} \mid\left\|z_{2}\right\|_{H^{1}\left(\Omega_{1}\right)}^{1 / 3} \\
& \leq C\left\|z_{2}\right\|_{H^{-2}\left(\Omega_{1}\right)}^{2 / 3}\left(\left\|z_{1}\right\|_{H^{1}\left(\Omega_{1}\right)}+\|z\|_{H^{1}\left(\Omega_{1}\right)}\right)^{1 / 3} \\
& \leq C\left\|z_{2}\right\|_{H^{-2}\left(\Omega_{1}\right)}^{2 / 3}\left(\varepsilon|\lambda|^{1 / 2}\|U\|_{\mathcal{H}}+C_{\varepsilon} \frac{1}{|\lambda|^{1 / 2}}\|F\|_{\mathcal{H}}\right)^{1 / 3} .
\end{aligned}
$$

Therefore, applying (3.25) into (3.27), we have

$$
\begin{aligned}
\left\|z_{2}\right\|_{H^{-1}\left(\Omega_{1}\right)} & \leq C\left(\frac{1}{|\lambda|}\|U\|_{\mathcal{H}}+\frac{C}{|\lambda|^{2}}\|F\|_{\mathcal{H}}\right)^{2 / 3}\left(\varepsilon|\lambda|^{1 / 2}\|U\|_{\mathcal{H}}+C_{\varepsilon} \frac{1}{|\lambda|^{1 / 2}}\|F\|_{\mathcal{H}}\right)^{1 / 3} \\
& \leq \varepsilon C \frac{1}{|\lambda|^{1 / 2}}\|U\|_{\mathcal{H}}+C_{\varepsilon} \frac{1}{|\lambda|^{3 / 2}}\|F\|_{\mathcal{H}}
\end{aligned}
$$

this is

$$
\left\|z_{2}\right\|_{H^{-1}\left(\Omega_{1}\right)} \leq \varepsilon C \frac{1}{|\lambda|^{1 / 2}}\|U\|_{\mathcal{H}}+C_{\varepsilon} \frac{1}{|\lambda|^{3 / 2}}\|F\|_{\mathcal{H}}
$$

Analogously we find

$$
\left\|y_{2}\right\|_{H^{-1}\left(\Omega_{2}\right)} \leq \varepsilon C \frac{1}{|\lambda|^{1 / 2}}\|U\|_{\mathcal{H}}+C_{\varepsilon} \frac{1}{|\lambda|^{3 / 2}}\|F\|_{\mathcal{H}}
$$

Also, applying (3.26) and Lemma 3.2. we have

$$
\left\|z_{2}\right\|_{H^{1}\left(\Omega_{1}\right)}+\left\|y_{2}\right\|_{H^{1}\left(\Omega_{2}\right)} \leq \varepsilon|\lambda|^{1 / 2}\|U\|_{\mathcal{H}}+C_{\varepsilon} \frac{1}{|\lambda|^{1 / 2}}\|F\|_{\mathcal{H}}
$$

Finally, using interpolation, the inequalities (3.28) and (3.30) lead to

$$
\begin{aligned}
\left\|z_{2}\right\|_{L^{2}\left(\Omega_{1}\right)} & \leq C\left\|z_{2}\right\|_{H^{-1}\left(\Omega_{1}\right)}^{1 / 2}\left\|z_{2}\right\|_{H^{1}\left(\Omega_{1}\right)}^{1 / 2} \\
& \leq C\left(\varepsilon \frac{1}{|\lambda|^{1 / 2}}\|U\|_{\mathcal{H}}+C_{\varepsilon} \frac{1}{|\lambda|^{3 / 2}}\|F\|_{\mathcal{H}}\right)^{1 / 2}\left(\varepsilon|\lambda|^{1 / 2}\|U\|_{\mathcal{H}}+C_{\varepsilon} \frac{1}{|\lambda|^{1 / 2}}\|F\|_{\mathcal{H}}\right)^{1 / 2} \\
& \leq C \varepsilon\|U\|_{\mathcal{H}}+C_{\varepsilon} \frac{1}{|\lambda|}\|F\|_{\mathcal{H}},
\end{aligned}
$$

or equivalently

$$
|\lambda|\left\|z_{2}\right\|_{L^{2}\left(\Omega_{1}\right)} \leq \varepsilon C|\lambda|\|U\|_{\mathcal{H}}+C_{\varepsilon}\|F\|_{\mathcal{H}} .
$$

Similarly, inequalities (3.29)-(3.30) lead to

$$
|\lambda|\left\|y_{2}\right\|_{L^{2}\left(\Omega_{2}\right)} \leq \varepsilon C|\lambda|\|U\|_{\mathcal{H}}+C_{\varepsilon}\|F\|_{\mathcal{H}} .
$$

As mentioned previously, $z_{1}$ and $y_{1}$ verify inequalities like (3.31) and (3.32), respectively. Therefore, we deduce that

$$
\begin{aligned}
|\lambda|^{2}\|z\|_{L^{2}\left(\Omega_{1}\right)}^{2} & \leq \varepsilon C|\lambda|^{2}\|U\|_{\mathcal{H}}^{2}+C_{\varepsilon}\|F\|_{\mathcal{H}}^{2} \\
|\lambda|^{2}\|y\|_{L^{2}\left(\Omega_{2}\right)}^{2} & \leq \varepsilon C|\lambda|^{2}\|U\|_{\mathcal{H}}^{2}+C_{\varepsilon}\|F\|_{\mathcal{H}}^{2}
\end{aligned}
$$


which leads to (3.18).

Finally, we estimate $|\lambda| \|\left.\Delta u\right|_{L^{2}\left(\Omega_{1}\right)}$ and $|\lambda||| \Delta v \|_{L^{2}\left(\Omega_{2}\right)}$ by the following Proposition.

Proposition 3.4. Let $U=(u, v, z, y, \theta, \omega)^{\prime} \in D(\mathcal{A})$ be a solution of the system (2.6)(2.11) with $F=\left(f^{1}, f^{2}, f^{3}, f^{4}, f^{5}, f^{6}\right)^{\prime} \in \mathcal{H}$. Then, for any $\varepsilon>0$, there exists $C_{\varepsilon}>0$ such that

$$
|\lambda|^{2}\|\Delta u\|_{L^{2}\left(\Omega_{1}\right)}^{2}+|\lambda|^{2}\|\Delta v\|_{L^{2}\left(\Omega_{2}\right)}^{2} \leq \varepsilon C|\lambda|^{2}\|U\|_{\mathcal{H}}^{2}+C_{\varepsilon}\|F\|_{\mathcal{H}}^{2} .
$$

Proof. Multiplying equation (2.8) by $z$ in $L^{2}\left(\Omega_{1}\right)$ and (2.9) by $y$ in $L^{2}\left(\Omega_{2}\right)$, we have

$$
\begin{array}{r}
i \lambda \rho_{1}\|z\|_{L^{2}\left(\Omega_{1}\right)}^{2}-\gamma_{1} \int_{\Gamma_{0}} \frac{\partial \Delta u}{\partial \nu} \bar{z} d \Gamma+\gamma_{1} \int_{\Gamma_{0}} \Delta u \frac{\partial \bar{z}}{\partial \nu} d \Gamma-\alpha_{1} \int_{\Gamma_{0}} \frac{\partial \theta}{\partial \nu} \bar{z} d \Gamma \\
-\alpha_{1} \int_{\Omega_{1}} \nabla \theta \nabla \bar{z} d x d y+\underbrace{\gamma_{1} \int_{\Omega_{1}} \Delta u \Delta \bar{z} d x d y}_{:=I_{1}}=\rho_{1} \int_{\Omega_{1}} f^{3} \bar{z} d x d y
\end{array}
$$

and

$$
\begin{array}{r}
i \lambda \rho_{2}\|y\|_{L^{2}\left(\Omega_{2}\right)}^{2}-\gamma_{2} \int_{\Gamma_{0}} \frac{\partial \Delta v}{\partial \nu} \bar{y} d \Gamma-\gamma_{2} \int_{\Gamma_{0}} \Delta v \frac{\partial \bar{y}}{\partial \nu} d \Gamma+\alpha_{2} \int_{\Gamma_{0}} \frac{\partial \omega}{\partial \nu} \bar{y} d \Gamma \\
-\alpha_{2} \int_{\Omega_{2}} \nabla \omega \nabla \bar{y} d x d y+\underbrace{\gamma_{2} \int_{\Omega_{2}} \Delta v \Delta \bar{y} d x d y}_{:=I_{2}}=\rho_{2} \int_{\Omega_{2}} f^{4} \bar{y} d x d y,
\end{array}
$$

respectively. Substituting $z$ given by (2.6) into $I_{1}$ and $y$ given by (2.7) into $I_{2}$ we deduce that

$$
\begin{array}{r}
i \lambda \rho_{1}\|z\|_{L^{2}\left(\Omega_{1}\right)}^{2}-i \lambda \gamma_{1}\|\Delta u\|_{L^{2}\left(\Omega_{1}\right)}^{2}-\int_{\Gamma_{0}}\left[\gamma_{1} \frac{\partial \Delta u}{\partial \nu}+\alpha_{1} \frac{\partial \theta}{\partial \nu}\right] \bar{z} d \Gamma+\gamma_{1} \int_{\Gamma_{0}} \Delta u \frac{\partial \bar{z}}{\partial \nu} d \Gamma \\
-\alpha_{1} \int_{\Omega_{1}} \nabla \theta \nabla \bar{z} d x d y=\rho_{1} \int_{\Omega_{1}} f^{3} \bar{z} d x d y+\gamma_{1} \int_{\Omega_{1}} \Delta u \Delta \overline{f^{1}} d x d y
\end{array}
$$

and

$$
\begin{array}{r}
i \lambda \rho_{2}\|y\|_{L^{2}\left(\Omega_{2}\right)}^{2}-i \lambda \gamma_{2}\|\Delta v\|_{L^{2}\left(\Omega_{1}\right)}^{2}+\int_{\Gamma_{0}}\left[\gamma_{2} \frac{\partial \Delta v}{\partial \nu}+\alpha_{2} \frac{\partial \omega}{\partial \nu}\right] \bar{y} d \Gamma+\gamma_{2} \int_{\Gamma_{0}} \Delta v \frac{\partial \bar{y}}{\partial \nu} d \Gamma \\
-\alpha_{2} \int_{\Omega_{2}} \nabla \omega \nabla \bar{y} d x d y=\rho_{2} \int_{\Omega_{2}} f^{4} \bar{y} d x d y+\gamma_{2} \int_{\Omega_{2}} \Delta v \Delta \overline{f^{2}} d x d y
\end{array}
$$

Adding equalities (3.33)-(3.34) and using transmission conditions (1.11)-(1.13), we obtain

$$
\begin{aligned}
&-i \lambda\left[\gamma_{1}\|\Delta u\|_{L^{2}\left(\Omega_{1}\right)}^{2}+\gamma_{2}\|\Delta v\|_{L^{2}\left(\Omega_{1}\right)}^{2}\right]=-i \lambda\left[\rho_{1}\|z\|_{L^{2}\left(\Omega_{1}\right)}^{2}+\rho_{2}\|y\|_{L^{2}\left(\Omega_{2}\right)}^{2}\right] \\
&+\alpha_{1} \int_{\Omega_{1}} \nabla \theta \nabla \bar{z} d x d y+\alpha_{2} \int_{\Omega_{2}} \nabla \omega \nabla \bar{y} d x d y+\rho_{1} \int_{\Omega_{1}} f^{3} \bar{z} d x d y+\gamma_{1} \int_{\Omega_{1}} \Delta u \Delta \overline{f^{1}} d x d y \\
&+\rho_{2} \int_{\Omega_{2}} f^{4} \bar{y} d x d y+\gamma_{2} \int_{\Omega_{2}} \Delta v \Delta \overline{f^{2}} d x d y
\end{aligned}
$$


Therefore, multiplying the last equality by $i \lambda$ and using Proposition 3.3 we obtain

$$
\begin{aligned}
|\lambda|^{2}\left[\gamma_{1}\|\Delta u\|_{L^{2}\left(\Omega_{1}\right)}^{2}+\gamma_{2}\|\Delta v\|_{L^{2}\left(\Omega_{1}\right)}^{2}\right] \leq & \varepsilon C|\lambda|^{2}\|U\|_{\mathcal{H}}^{2}+C_{\varepsilon}\|F\|_{\mathcal{H}}^{2} \\
& +C|\lambda|\|\nabla \theta\|_{L^{2}\left(\Omega_{1}\right)}\|\nabla z\|_{L^{2}\left(\Omega_{2}\right)} \\
& +C|\lambda|\|\nabla \omega\|_{L^{2}\left(\Omega_{1}\right)}\|\nabla y\|_{L^{2}\left(\Omega_{2}\right)} .
\end{aligned}
$$

Note that, by Lemmas 2.3 and 3.2, we have

$$
\begin{aligned}
|\lambda||| \nabla \theta\left\|_{L^{2}\left(\Omega_{1}\right)}|| \nabla z\right\|_{L^{2}\left(\Omega_{2}\right)} & \leq \varepsilon C|\lambda|^{2}|| U\left\|_{\mathcal{H}}^{2}+C_{\varepsilon}\right\| F \|_{\mathcal{H}}^{2} \\
|\lambda|\|\nabla \omega\|_{L^{2}\left(\Omega_{1}\right)}|| \nabla y \|_{L^{2}\left(\Omega_{2}\right)} & \leq \varepsilon C|\lambda|^{2}|| U\left\|_{\mathcal{H}}^{2}+C_{\varepsilon}\right\| F \|_{\mathcal{H}}^{2} .
\end{aligned}
$$

Finally inserting these inequalities into (3.35), our conclusion follows.

We are now in position to establish the main theorem of this paper.

Theorem 3.5. The semigroup generated by the linear operator $\mathcal{A}$ given by (2.2) and associated to system (1.6)-(1.13) is of analytic type.

Proof. By Theorem 2.2 it is enough to show that the operator $\mathcal{A}$ verifies conditions (2.3)-(2.4). It's not difficult to verify condition (2.3) (see [6]). In order to verify condition (2.4), let $U \in D(\mathcal{A})$ and $F \in \mathcal{H}$ such that

$$
(i \lambda I-\mathcal{A}) U=F \quad \text { in } \quad \mathcal{H} .
$$

Then, by Propositions $3.1,3.3$ and 3.4, we obtain

$$
|\lambda|^{2}|| U\left\|_{\mathcal{H}}^{2} \leq \varepsilon C|\lambda|^{2}\right\| U\left\|_{\mathcal{H}}^{2}+C_{\varepsilon}\right\| F \|_{\mathcal{H}}^{2} .
$$

Choosing $\varepsilon$ small enough, we deduce that there exists $M>0$ such that

$$
|\lambda||| U\left\|_{\mathcal{H}} \leq M|| F\right\|_{\mathcal{H}}
$$

this is

$$
\left\|\lambda(i \lambda I-\mathcal{A})^{-1}\right\|_{\mathcal{L}(\mathcal{H})} \leq M, \quad \forall|\lambda| \geq 1
$$

Then

$$
\lim _{|\lambda| \rightarrow \infty}\left\|\lambda(i \lambda I-\mathcal{A})^{-1}\right\|_{\mathcal{L}(\mathcal{H})}<\infty
$$

which completes the proof.

\section{REFERENCES}

[1] Kim, J. U.: On the energy decay of a linear thermoelastic bar and plate. SIAM J. Math. Anal. 23 (1992), 889-899. MR.1166563 (93f:35159)

[2] Lagnese, J. E.: Boundary Stabilization of Thin Plates. SIAM, Philadelphia (1989). MR1061153 (91k:73001)

[3] Lasiecka, I., Triggiani, R.: Analyticity, and lack thereof, of thermo-elastic semigroups. ESAIM: Proceedings, Contrôle et Équations aux Dérivées Partielles. 4 (1998), 199-222. MR.1663662 (99m:35243)

[4] Liu, K., Liu, Z.: Exponential stability and analyticity of abstract linear thermoelastic systems. Z. Angew. Math. Phys. 48 (1997), 885-904. MR1488686 (98m:35207)

[5] Liu, Z. Y., Renardy, M.: A note on the equation of a thermoelastic plate. Appl. Math. Lett. 8 (1995), 1-6. MR 1356798 (96i:35128)

[6] Liu, Z., Zheng, S.: Semigroups associated with dissipative systems. Research Notes Math. 398, Chapman \& Hall/CRC, Boca Raton (1999). MR.1681343 (2000c:47080) 
[7] Muñoz Rivera, J. E., Shibata, Y.: A linear thermoelastic plate equation with Dirichlet boundary conditions. Math. Meth. Appl. Sci. 20 (1997), 915-932. MR1458525 (98d:73010)

[8] Muñoz Rivera, J. E., Fatori, L. H.: Regularizing properties and propagations of singularities for thermoelastic plates. Math. Meth. Appl. Sci. 21 (1998), 797-821. MR1626986 (99f:73013)

[9] Muñoz Rivera, J. E., Portillo Oquendo, H.: A transmission problem for thermoelastic plates. Quarterly of Applied Mathematics. 62(2) (2004), 273-293. MR2054600 (2005b:74035)

[10] Pazy, A.: Semigroup of linear operators and applications to partial differential equations. SpringerVerlag, New York, 1983. MR.710486 (85g:47061)

[11] Shibata, Y.: On the exponential decay of the energy of a linear thermoelastic plate. Comp. Appl. Math. 13 (1994), 81-102. MR 1361587 (96k:35178) 